09

\title{
Взаимодействие прямоугольного униполярного импульса С двухуровневой резонансной средой
}

\author{
(C) P.М. Архипов ${ }^{1,2}$, Н.Н. Розанов ${ }^{2}$ \\ ${ }^{1}$ Санкт-Петербургский государственный университет, \\ 199034 Санкт-Петербург, Россия \\ ${ }^{2}$ ФТИ им. А.Ф. Иофффе, \\ 194021 Санкт-Петербург, Россия \\ e-mail: arkhipovrostislav@gmail.com
}

Поступила в редакцию 21.01.2020 г.

В окончательной редакции 21.01.2020 г.

Принята к публикации 27.01.2020 г.

Интерес к униполярным импульсам в оптике связан с возможностью их эффективного воздействия на квантовые объекты. В работе изучается взаимодействие короткого прямоугольного униполярного импульса с двухуровневой резонансной средой в условиях, когда длительность импульса меньше времен релаксации среды. Парадоксальным является тот факт, что поляризация и разность заселенностей среды осциллирует не на частоте Раби, а на частоте, пропорциональной сумме квадратов частоты резонансного перехода среды и частоты Раби импульса. Данное явление аналогично эффекту Штарка, которое заключается в сдвиге частот переходов среды в постоянном внешнем электрическом поле. Обсуждается возможность генерации излучения на частотах, превосходящих частоту резонансного перехода при возбуждении среды прямоугольным униполярным импульсом.

Ключевые слова: униполярные импульсы, двухуровневая система, осцилляции Раби.

DOI: $10.21883 /$ OS.2020.05.49323.9-20

\section{Введение}

В последнее время в литературе обсуждается возможность получения коротких электромагнитных импульсов с высокой степенью униполярности, определяемой как (см. обзоры [1-3] и цитируемую литературу).

$$
\xi=\frac{\left|\int E d t\right|}{\int|E| d t} .
$$

В числителе (1) стоит электрическая площадь электромагнитного импульса, которая определяется выражением [4]

$$
S_{E}=\int_{t=-\infty}^{+\infty} E(t) d t,
$$

где $E(t)$ - напряженность электрического поля и $t-$ время. Классические биполярные импульсы обладают нулевой площадью и степенью униполярности. На практике удается получить квазиуниполярные предельно короткие импульсы полуцикловой формы, т. е. содержащие всплекс поля большой амплитуды и длинный затухающий хвост противоположной полярности, в ТГц и оптическом диапазоне [5-11]. Полуцикловая форма таких импульсов позволяет говорить о возможности однонаправленного воздействия на микрообъекты и эффективном использовании таких импульсов для возбуждения и контроля динамики волновых пакетов [2,3,12-16], а также для ускорения заряженных частиц [17]. Поэтому изучение взаимодействия униполярных импульсов с веществом является актуальной проблемой. В предыдущих исследованиях, в основном изучались вопросы эффективности воздействия униполярных импульсов на квантовые объекты [2-3,14], возможность селективного воздействия на резонансные переходы в атомах $[15,16]$ и ряд вопросов, связанных с распространением полуцикловых импульсов в резонансных средах [4]. А ряд вопросов динамики системы под действием униполярных остаются под вопросом.

Как известно, при когерентном взаимодействии резонансного излучения с двухуровневой средой (когда частота Раби больше обратного времени релаксации поляризации среды, $\Omega_{R}>\frac{1}{T_{2}}$, и длительность импульса излучения короче времени релаксации поляризации, $\tau<T_{2}$ ) атомная поляризация и инверсия осциллируют с частотой Раби (Раби осцилляции) [18]. В случае униполярных импульсов взаимодействие таких импульсов со средой имеет нерезонансный характер. Поэтому интересным является вопрос о поведении двухуровневой среды в поле униполярного импульса. В последнее время появилось большое число работ, в которых предложены способы получения квазиуниполярных импульсов прямоугольной формы [19-27] в различных спектральных диапазонах частот. Целью данной работы является изучение взаимодействия прямоугольного униполярного импульса с двухуровневой резонансной средой. Показана на первый взгляд необычная возможность сверхбыстрых осцилляций атомной инверсии и поляризации не на частоте Раби, а на частоте, пропорционалной сумме квад- 

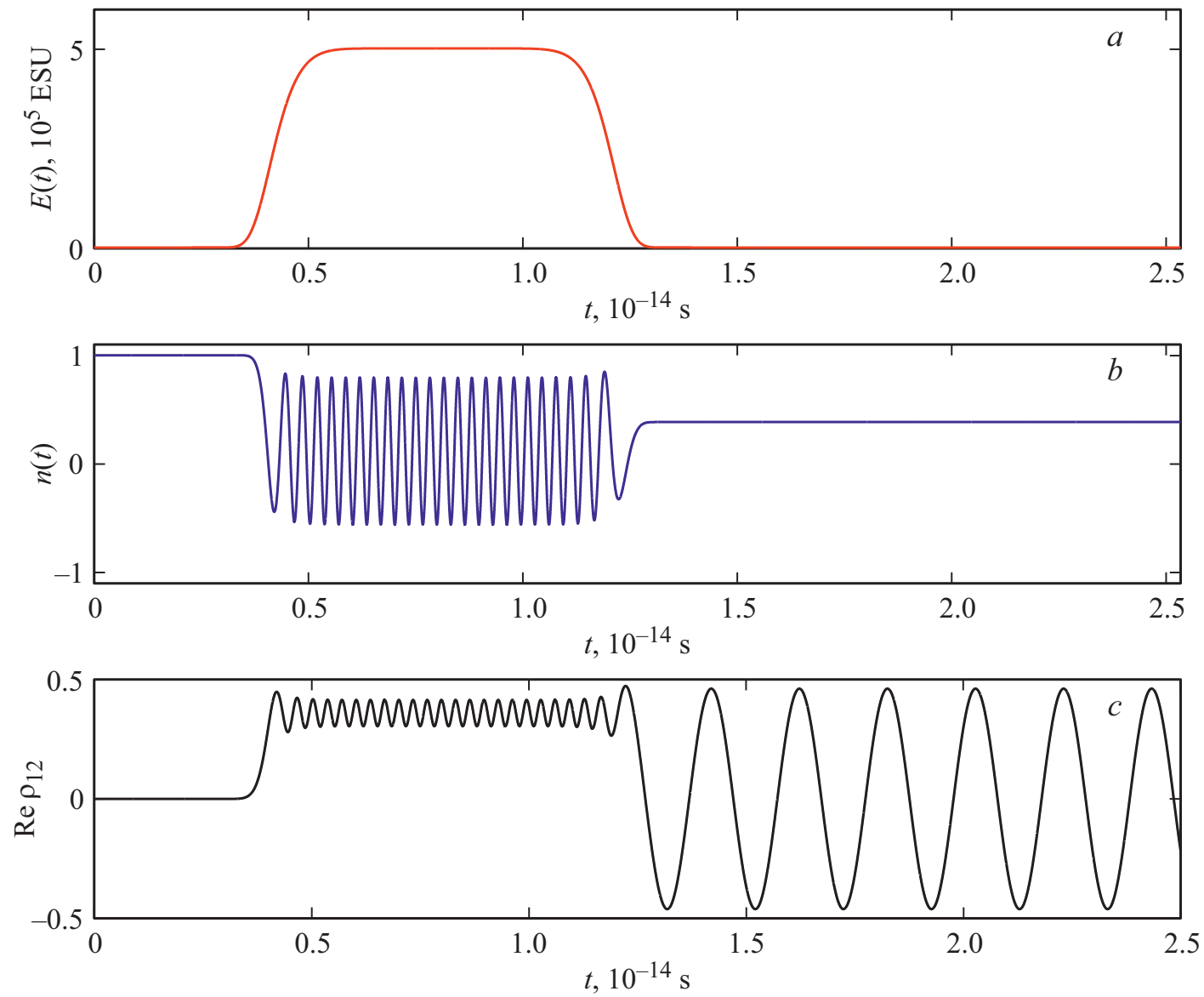

Рис. 1. Временное поведение электрического поля прямоугольного импульса $E(t)(a)$, разности заселенностей $n(t)(b)$ и вещественной части недиагонального элемента матрицы плотности $\operatorname{Re} \rho_{12}(c)$. Параметры расчета указаны в таблице.

ратов частоты Раби и частоты резонансного перехода среды. Обсуждается возможность генерации излучения на частоте выше частоты резонанса среды.

\section{Взаимодействие двухуровневой среды с прямоугольным импульсом}

Взаимодействие двухуровневой резонансной среды с электрическим полем короткого импульса описывается системой уравнений для матрицы плотности, которая без учета релаксации среды имеет вид $[28,29]$

$$
\begin{gathered}
\frac{\partial \rho_{12}(t)}{\partial t}=i \omega_{0} \rho_{12}(t)-\frac{i}{\hbar} d_{12} E(t) n(t), \\
\frac{\partial n(t)}{\partial t}=\frac{4}{\hbar} d_{12} E(t) \mathscr{Y} \rho_{12}(z, t), \\
P(t)=2 d_{12} \mathscr{R} \rho_{12} .
\end{gathered}
$$

В этих уравнениях $\rho_{12}-$ недиагональный элемент матрицы плотности, $n \equiv \rho_{11}-\rho_{22}-$ разность заселенностей (инверсия) между основным и возбужденным состояниями двухуровневой системы, $P-$ поляризация единичного атома, $c$ - скорость света в вакууме, $\hbar$ - приведенная постоянная Планка, $\omega_{0}$ - частота резонансного перехода среды, $d_{12}$ - дипольный момент перехода.

Нетрудно показать, что система уравнений Блоха (3), (4) может быть сведена к уравнениям для поляризации $P$ и разности заселенностей $n$ в форме [29]:

$$
\begin{gathered}
\ddot{P}+\omega_{0}^{2} P=\frac{2 \omega_{0} d_{12}^{2}}{\hbar} E(t) n(t), \\
\dot{n}=-\frac{2}{\hbar \omega_{0}} E(t) \dot{P}(t) .
\end{gathered}
$$

Пусть теперь на систему действует прямоугольный импульс амплитуды $E_{0}$ и длительности $\tau$. Будем считать, что длительность импульса существенно меньше времен релаксации разности заселенностей $T_{1}$ и поляризации $T_{2}$ среды, т. е. импульс взаимодействует со средой когерентно. Поэтому времена релаксации можно считать бесконечно большими. Тогда за время действия импульса в правых частях уравнений $(6),(7)$ поле $E(t)$ можно заменить на постоянную величину $E_{0}$ :

$$
\ddot{P}+\omega_{0}^{2} P=\frac{2 \omega_{0} d_{12}^{2}}{\hbar} E_{0} n(t)
$$



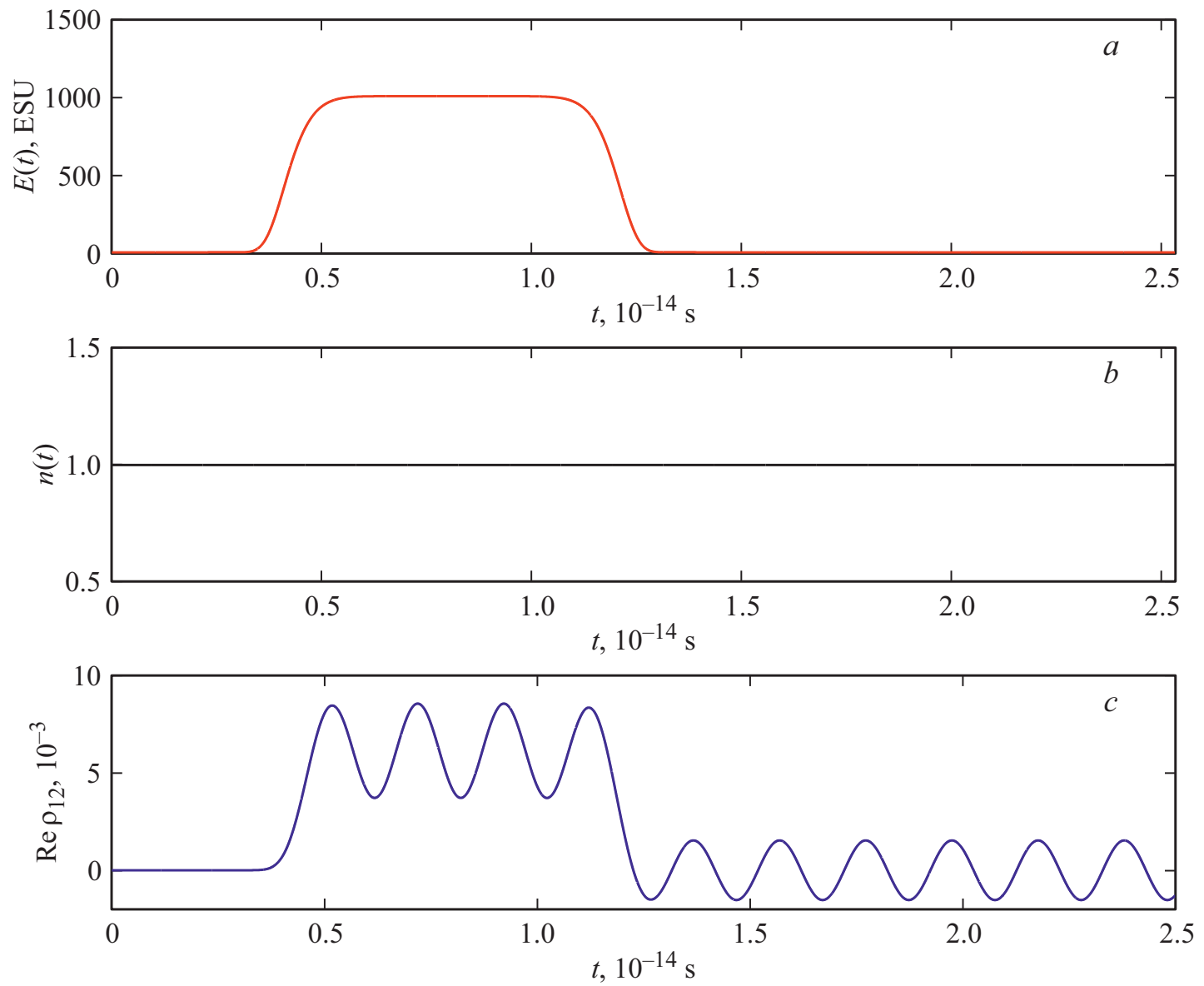

Рис. 2. Временное поведение электрического поля прямоугольного импульса $E(t)(a)$, разности населенностей $n(t)(b)$ и вещественной части недиагонального элемента матрицы плотности Re $\rho_{12}(c)$. Амплитуда поля $E_{0}=1000 \mathrm{ESU}$. Частота Раби $\Omega_{R}=1.9 \cdot 10^{13} \mathrm{rad} / \mathrm{s}$. Остальные параметры расчета указаны в таблице.

Параметры модели, используемые в расчетах

\begin{tabular}{|c|c|}
\hline Амплитуда импульса & $E_{0}=5 \cdot 10^{5} \mathrm{ESU}$ \\
\hline Параметр $\tau$ & $\tau=4 \mathrm{fs}$ \\
\hline Частота перехода среды & $\omega_{0}=3.1 \cdot 10^{15} \mathrm{rad} / \mathrm{s}$ \\
\hline Дипольный момент перехода & $d_{12}=20 \mathrm{D}$ \\
\hline Частота Раби & $\Omega_{R}=9.5 \cdot 10^{15} \mathrm{rad} / \mathrm{s}$ \\
\hline $\begin{array}{c}\text { Частота осцилляций поляризации и } \\
\text { разности населенностей }\end{array}$ & $\Omega=1.9 \cdot 10^{16} \mathrm{rad} / \mathrm{s}$ \\
\hline Времена релаксации & $T_{1}=T_{2}=\infty$ \\
\hline
\end{tabular}

Интегрируя уравнение (9) по времени от 0 до $t$ (рассматриваем интервал времени действия импульса $0 \leq t \leq \tau)$, с учетом начальных условий $n(0)=1$, $P(0)=0, \dot{P}(0)=0$, получаем для инверсии

$$
n(t)=1-\frac{2}{\hbar \omega_{0}} E_{0} P(t)
$$

Подставляя (10) в уравнение (8), получаем уравнение для поляризации

$$
\ddot{P}+\Omega^{2} P=\frac{2 \omega_{0} d_{12}^{2}}{\hbar} E_{0},
$$

где введена новая частота $\Omega^{2} \equiv \omega_{0}^{2}+4 \Omega_{R}^{2}, \Omega_{R}=\frac{d_{12} E_{0}}{\hbar}-$ частота Раби импульса возбуждения. Из (11) видно, что атомная поляризация в сильном поле прямоугольного импульса будет совершать осцилляции на новой частоте $\Omega$, которая выше частоты перехода.

Решения уравнений (8) и (9) для атомной поляризации $P$ и разности заселенностей $n$ (при начальных условиях $n(0)=1, P(0)=0, \dot{P}(0)=0)$ за время действия импульса при $0 \leq t \leq \tau$ имеют вид

$$
\begin{gathered}
n(t)=\frac{4 \Omega_{R}^{2} \cos (\Omega t)+\omega_{0}^{2}}{\omega_{0}^{2}+4 \Omega_{R}^{2}}, \\
P(t)=\frac{2 \omega_{0} d_{12}^{2} E_{0}}{\hbar\left(\omega_{0}^{2}+4 \Omega_{R}^{2}\right)}[1-\cos (\Omega t)] .
\end{gathered}
$$

Из (12) вытекает на первый взгляд парадоксальный факт, что под действием сильного поля прямоугольного 
импульса атомная поляризация и инверсия за время действия импульса совершают колебания на высокой частоте $\Omega \equiv \sqrt{\omega_{0}^{2}+4 \Omega_{R}^{2}}$, а не на частоте Раби, как это происходит при воздействии на двухуровневую среду биполярного импульса или монохроматического излучения [18]. Однако данный парадокс снимается, если вспомнить, что в постоянном поле происходит сдвиг частоты атомного перехода (эффект Штарка) на величину, в первом приближении пропорциональную квадрату напряженности внешнего поля (для неводородоподобных атомов) [30]. Данная поляризация может являться источником излучения на частоте $\Omega$, выше частоты излучения среды. Поэтому интересным является вопрос о применении униполярных импульсов для генерации или преобразования частоты резонансного излучения среды. В частности, если частота Раби удовлетворяет условию $\Omega_{R}=\sqrt{m^{2}-1} \frac{\omega_{0}}{2}$, где $m=2,3,4 \ldots$, то возможна генерация гармоник частоты перехода среды.

Сказанное проиллюстрируем численным расчетом. На рис. 1 представлено временное поведение электрического поля прямоугольного импульса $E(t)(a)$, разности заселенностей $n(t)(b)$ и вещественной части недиагонального элемента матрицы плотности $\operatorname{Re} \rho_{12}(c)$. Данные зависимости были получены в результате численного решения системы уравнений для матрицы плотности (3), (4). Поле прямоугольного импульса аппроксимировалось гипергауссовой функцией $E(t)=E_{0} e^{-\frac{t^{10}}{\tau^{10}}}$. Не останавливаясь на вопросах конкретизации типа резонансных сред, применимости двухуровневого приближения и пр., для наглядной иллюстрации исследуемого явления примем модельные параметры, которые указаны в таблице.

Из рис. 1 видно, что поляризация и инверсия осциллируют на частоте $\Omega$ за время действия импульса. При указанных параметрах период этих осцилляций состаляет $T=0.32 \mathrm{fs}$, что примерно в 6 раз меньше периода резонансного перехода $T_{0}=2 \mathrm{fs}$. После окончания действия импульса поляризация среды осциллирует на частоте перехода $\omega_{0}$. Аналогичные осцилляции населенностей, как показывают расчеты, наблюдаются и в трехуровневой схеме, но исследования данного вопроса выходят за рамки данной работы.

В случае слабого поля при $\Omega_{R} \rightarrow 0$, как следует из $(12)$, инверсия $n \rightarrow 1$, т. е. не меняется, как и должно быть в случае слабого поля. А поляризация будет осциллировать на частоте перехода. Данное обстоятельство подтверждается численным расчетом, (рис. 2).

\section{Заключение}

Таким образом, в работе изучены особенности взаимодействия униполярного импульса прямоугольной формы с двухуровневой резонансной средой. В случае резонансного воздействия коротких биполярных импульсов на двухуровневую среду, т.е. при когерентном взаимодействии импульса со средой, атомная поляризация и разность заселенностей осциллируют с частотой Раби. В случае же униполярного импульса ситуация иная, взаимодействие со средой имеет нерезонансный характер. Показано, что в сильном поле прямоугольного импульса, поляризация и инверсия осциллируют не на частоте Раби, а на частоте, пропорциональной сумме квадратов частот перехода среды и частоты Раби, $\Omega \equiv \sqrt{\omega_{0}^{2}+4 \Omega_{R}^{2}}$. Если амплитуда прямоугольного импульса мала, то инверсия среды не меняется, а поляризация осциллирует на частоте перехода. Появление новой частоты $\Omega$ аналогично эффекту Штарка, который заключается в сдвиге частот переходов среды в постоянном внешнем электрическом поле [30,31].

Осциллируюшая поляризация среды на частоте $\Omega$ может являться источником когерентного излучения на частоте, превышающей частоту перехода. Поэтому изученное явление наводит на мысль об использовании униполярных импульсов для генерации электромагнитного излучения, частотой которого $\Omega$ можно управлять с помощью изменения частоты Раби возбуждающего импульса, и в частности для генерации гармоник частоты перехода среды.

Отметим, что различные способы получения квазиуниполярных импульсов прямоугольной формы в ТГц и оптическом диапазоне частот были недавно предложены в работах [19-27]. Хоть униполярные импульсы и кажутся экзотическими на сегодняшний день, исследованный эффект еще раз показывает возможность применения униполярных импульсов для сверхбыстрого воздействия на резонансные среды, как отмечалось в работах [13,13-17].

\section{Финансирование работы}

Аналитические исследования взаимодействия прямоугольного импульса с двухуровневой резонансной средой выполнены при финансовой поддержке РФФИ в рамках научного проекта № 20-32-70049. Численное моделирование выполнено при финансовой поддержке РНФ, проект № 17-19-01097.

\section{Конфликт интересов}

Авторы заявляют, что у них нет конфликта интересов.

\section{Список литературы}

[1] Архипов Р.М., Пахомов А.В., Архипов М.В., Бабушкин И., Толмачев Ю.А., Розанов Н.Н. // Письма в ЖЭТФ. 2017. T. 105. № 6. C. 388 (2017); Arkhipov R.M., Pakhomov A.V., Babushkin I., Tolmachev Yu.A., Rosanov N.N. // JETP Letters. 2017. V. 105. N 6. P. 408.

[2] Розанов Н.Н., Архипов М.В., Архипов Р.М., Веретенов Н.А., Пахомов А.В., Федоров С.В. // Опт. и спектр. 2019. T. 127. Вып. 7. C. 82; Rosanov N.N., Arkhipov M.V., Arkhipov R.M., Veretenov N.A., Pakhomov A.V., Fedorov S.V. // Opt. Spectr. 2019. V. 127. N 1. P. 77. 
[3] Архипов Р.М., Архипов М.В., Шимко А.А., Пахомов А.В., Розанов Н.Н. // Письма в ЖЭТФ. 2019. Т. 110. № 1. С. 9; Arkhipov R.M. Arkhipov M.V., Shimko A.A., Rosanov N.N. // JETP Lett. 2019. V. 110. N 1. P. 15.

[4] Розанов Н.Н., Архипов Р.М., Архипов М.В. // УФН. 2018. T. 188. № 12. C. 1347; Rosanov N.N., Arkhipov R.M., Arkhipov M.V. // Phys. Usp. 2018. V. 61. N 12. P. 1227.

[5] Hassan M.T., Luu T.T., Moulet A., Raskazovskaya O. et al. // Nature. 2016. V. 530. P. 66.

[6] Wu H.-C., Meyer-ter-Vehn J. // Nature Photon. 2012. V. 6. P. 304.

[7] Xu J., Shen B., Zhang X. et al. // Sci. Rep. 2018. V. 8. P. 2669.

[8] Reiman K. // Rep. Progr. Phys. 2007. V. 70. P. 1597.

[9] Roskos H.G., Thomson M.D., Kress M., Loeffler T. // Laser Photon. Rev. 2007. V. 1. P. 349.

[10] Gao Y., Drake T., Chen Z., DeCamp M.F. // Opt. Lett. 2008. V. 33. P. 2776.

[11] Obraztsov P.A., Kaplas T., Garnov S.V., Kuwata-Gonokami M., Obraztsov A.N., Svirko Y.P. // Sci. Reports. 2014. V. 4. P. 4007.

[12] Wetzels A., Gjrtler A., Noordam L.D., Robicheaux F., Dinu C., Muller H.G., Vrakking M.J., van der Zande W.J. // Phys. Rev. Lett. 2002. V. 89. P. 273003.

[13] Chai X., Ropagnol X., Mohsen Raeis-Zadeh S., Reid M., Safavi-Naeini S., Ozaki T. // Phys. Rev. Lett. 2018. V. 121. P. 143901.

[14] Arkhipov R.M., Arkhipov M.V., Babushkin I.V., Demircan A., Morgner U., Rosanov N.N. // Opt. Lett. 2019. V. 44. N 5. P. 1202.

[15] Архипов Р.М., Архипов М.В., Пахомов А.В., Розанов Н.Н. // Квант. электрон. 2019. Т. 49. № 10. С. 958-962; Arkhipov R.M., Arkhipov M.V., Pakhomov A.V., Rosanov N.N. // Quantum Electron. 2019. V. 49. N 10. P. 958-962.

[16] Архипов Р.М., Архипов М.В., Пахомов А.В., Розанов Н.Н. // Опт. и спектр. 2020. Т. 128. № 1. С. 106-109; Arkhipov R.M., Arkhipov M.V., Pakhomov A.N., Rosanov N.N. // Opt. Spectrosc. 2020. V. 128. N 1. P. 102-105.

[17] Розанов Н.Н., Высотина Н.В. // ЖЭТФ. 2020. Т. 157. В. 1. C. 63.

[18] Аллен Л., Эберли Дж. Оптический резонанс и двухуровневые атомы. М.: Мир, 1978; Allen L., Eberly J.H. Optical Resonance and Two-Level Atoms. N.Y.: Wiley, 1975.

[19] Arkhipov R.M., Arkhipov M.V., Belov P.A., Tolmachev Yu.A., Babushkin I. // Las. Phys. Lett. 2016. V. 13. P. 046001.

[20] Arkhipov R.M., Pakhomov A.V., Babushkin I.V., Arkhipov M.V., Tolmachev Yu.A., Rosanov N.N. // J. Opt. Soc. Am. B. 2016. V. 33. P. 2518.

[21] Pakhomov A.V., Arkhipov R.M., Babushkin I.V., Arkhipov M.V., Tolmachev Yu.A., Rosanov N.N. Phys. Rev. A. 2017. V. 95. P. 013804.

[22] Ziguleva D.O., Arkhipov R.M., Arkhipov M.V., Pakhomov A.V., Babushkin I., Rosanov N.N. // Opt. Commun. 2016. V. 424. P. 170-176

[23] Pakhomov A.V., Arkhipov R.M., Arkhipov M.V., Demircan A., Morgner U., Rosanov N.N. // Sci. Rep. 2019. V. 9. N 1. P. 7444.

[24] Sazonov S.V., Ustinov N.V. Phys. Rev. A. 2018. V. 98. P. 063803.

[25] Bakunov M.I., Maslov A.V., Tsarev M.V. // Phys. Rev. A. 2017. V. 95. N 6. P. 063817.

[26] Efimenko E.S., Sychugin S.A., Tsarev M.V., Bakunov M.I. // Phys. Rev. A. 2018. V. 98. N 1. P. 01384.

[27] Tsarev M.V., Bakunov M.I. // Opt. Express. 2019. V. 27. N 4. P. 5154-5164.
[28] Ярив А. Квант. электрон. М.: Сов.радио. 1980. Yariv A. Quantum Electronics. Wiley, 1989.

[29] Ахманов С.А., Никитин С.Ю. Физическая оптика. М.: Наука, 2004; Akhmanov S.A., Nikitin S.Y. Physical Optics. Clarendon Press, 1997.

[30] Ландау Л.Д., Лифиии, Е.М. Квантовая механика. Нерелятивистская теория. М.: Наука, 1989. 768 с.; Landau L.D., Lifshitz E.M. Quantum Mechanics. Pergamon, 1974.

[31] Фриш С.Э. Оптические спектры атомов. Москва-Ленинград: Государственное издательство физико-математической литературы, 1963. 